\title{
Is there a role of fortified food in maintaining vitamin B12 status in proton pump inhibitor users?
}

\author{
C. F. Hughes ${ }^{1}$, M. Ward ${ }^{1}$, L. Hoey ${ }^{1}$, K. Pentieva ${ }^{1}$, A. Molloy ${ }^{2}$, J. M. Scott ${ }^{2}$, F. Tracey ${ }^{3}$ \\ and H. McNulty ${ }^{1}$ \\ ${ }^{1}$ Northern Ireland Centre for Food and Health, University of Ulster, Coleraine, BT52 1SA, ${ }^{2}$ Trinity College, \\ School of Biochemistry and Immunology, Dublin, Ireland and ${ }^{3}$ Causeway Hospital, Coleraine, BT52 HIS, UK
}

Proton pump inhibitor (PPI) drugs are potent gastric acid suppressant medications used for the treatment of a variety of gastric-related disorders. PPIs induce a state clinically identical to atrophic gastritis, a leading cause of food-bound malabsorption of vitamin B12. Considering that absorption of food-bound vitamin B12 is dependent on gastric acid, there is a concern that long-term use of PPI drugs may lead to vitamin B12 depletion, however, the evidence is conflicting ${ }^{(1,2)}$. Theoretically, the absorption of free vitamin B12 should continue undisrupted with PPI usage because it has no gastric acid requirement. The aim of this study was to examine the potential role of fortified food in preventing any depletion in vitamin B12 status owing to long-term usage of PPIs. Fortified food consumption and B-vitamin status were assessed in PPI users and in age-matched adults who were non-PPI users. Patients who had received PPI maintenance therapy for a period of $>1 \mathrm{y}$ were identified from a local GP practice, while non-PPI users were identified from a previous study to investigate B-vitamin intake and status in healthy male and female adults ${ }^{(3)}$.

\begin{tabular}{|c|c|c|c|c|c|c|c|c|c|c|}
\hline & & & & & & & Non & Jsers & & \\
\hline & & CoI & FF & & & & Col & FF & & \\
\hline & & & & & & & & & & \\
\hline & & & & & $\boldsymbol{P}$ & & & & & $P$ \\
\hline Age (Yr) & 56.0 & $(16.0)$ & 56.0 & $(26.0)$ & 0.318 & 56.0 & $(16.0)$ & 56.5 & $(19.0)$ & 0.421 \\
\hline Total B12 Intake $(\mu \mathrm{g} / \mathrm{d})$ & 3.4 & $(2.7)$ & 3.9 & $(1.8)$ & 0.458 & 3.6 & $(2.1)$ & 3.2 & $(2.5)$ & 0.028 \\
\hline $\mathrm{RCF}(\mathrm{nmol} / \mathrm{l})$ & 909 & (739) & 590 & $(383)$ & $<0.001$ & 929 & $(520)$ & 713 & (364) & $<0.001$ \\
\hline $\mathrm{SF}(\mathrm{nmol} / \mathrm{l})$ & 15.5 & $(17.8)$ & 10.6 & $(10.5)$ & 0.019 & 23.1 & (16.7) & 18.1 & $(13.0)$ & $<0.001$ \\
\hline Serum B12 (pmol/l) & 366 & (114) & 256 & (144) & 0.001 & 282 & (142) & 242 & (112) & 0.234 \\
\hline Serum HoloTC $(\mathrm{pmol} / \mathrm{l})$ & 59.6 & $(34.2)$ & 38.5 & $(23.9)$ & 0.001 & 53.9 & $(29.1)$ & 48.2 & $(26.5)$ & 0.559 \\
\hline
\end{tabular}

Values are presented as median (IQR). Differences between consumers and non consumers were assessed using an independent t-test for continuous variables (following logtransformation of data as appropriate). Abbreviations: FF, fortified food consumer; Hcy, homocysteine; RCF, red cell folate; SF, serum folate; holoTC, holotranscobalamin. Participants were classified as fortified food consumers if they consumed foods fortified with B-vitamins at least once per week.

Regular consumption of fortified food was associated with significantly greater folate status (i.e. serum and red cell folate concentrations) among both the PPI users and non-users. In contrast, consumption of fortified foods had a significant impact on vitamin B12 status only in patients on PPI drugs (as measured by two biomarkers, serum total vitamin B12 and holoTC) but had no effect in the non-PPI users. Furthermore, a significantly greater proportion of subclinical deficiency (serum B $12<150$ pmol/l) was observed among the long-term PPI users who did not regularly consume food fortified foods $(14 \%)$ compared to consumers (1\%; chi-square; $P=0.044)$. The current study demonstrates that consumption of fortified foods appears to protect against vitamin B12 depletion in patients on long-term PPI therapy. However, at current levels of fortification, the consumption of fortified foods in adults generally (not taking PPI drugs) was not associated with improved vitamin B12 status.

1. Dharmarajan TS, Kanagala MR, Murakonda P et al. (2008) JAMDA 9, 162-167.

2. den Elzen WP, Groeneveld Y, de Ruijter, W et al. (2008) Aliment Pharmacol Ther 27, 491-497.

3. Hoey L, McNulty H, Askin N et al. (2007) Am J Clin Nutr 86, 1405-1413. 